\title{
DOE/ER/14181-TT)
}

\section{Theoretical Treatment of the Thermophysical Properties of Fluids Containing Chain-like Molecules}

Final Technical Report Submitted to The Department of Energy

Contract Number DE-FG05-91ER14181

by

Carol K. Hall

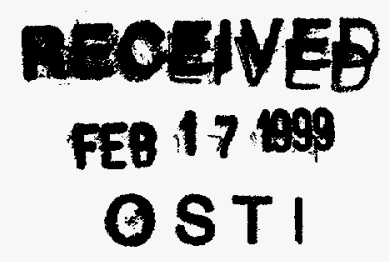

Department of Chemical Engineering

North Carolina State University

Raleigh, North Carolina 27695-7905

June 1, 1994 - May 31, 1997

DISTABUTION OF THIS DOCUMENT IS LARMITED

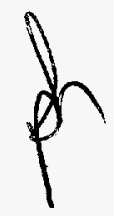




\section{DISCLAIMER}

This report was prepared as an account of work sponsored by an agency of the United States Government. Neither the United States Government nor any agency thereor, aor any of their exployees, makes any warranty, express or implied, or assumes any legal liability or responsibility for the accurncy, completeness, or usefulness of any information, apparatus, product, or process disclosed, or represents that its use would not infringe privately owned rights. Refereace herein to any specific commercial product, process, or service by trade name, trademart, inanufacturer, or otherwise does not necessarily constitute or imply its endorsement, recommendation. or favoring by the United States Government or any agency thereof. The views and opinions of authors expressed herein do not necessarily state or reflect those of the United States Government or any agency thereof. 


\section{DISCLAIMER}

Portions of this document may be illegible in electronic image products. Images are produced from the best available original document. 


\title{
Theoretical Treatment of the Thermophysical Properties of Fluids \\ Containing Chain-like Molecules
}

\author{
Progress Report \\ December, 1993 - December, 1996
}

\section{Introduction}

We have been engaged in a research program aimed at enhancing our understanding of the thermophysical properties of fluids containing long, flexible, chain-like molecules. We have been working on four main fronts: (1) the development of an equation of state that is capable of predicting the experimentally observed thermodynamic properties, including phase equilibria, of fluids containing chain-like molecules ranging in length from alkanes to polymers, (2) computer simulation studies of the transport properties of chain fluids, with special focus on the role played by entanglements in the dynamical properties of polymer melts, (3) computer simulation studies and theoretical treatment of the static and dynamic properties of polymer networks and gels, and (4) computer simulation studies of the permeation of penetrants in polymer membranes. The theories resulting from this research could eventually serve as the foundation upon which to build correlations of petroleum and natural gas, as well as of polymer solutions, melts, blends, networks, and gels.

The theoretical foundation for our work is the Generalized Flory-Dimer (GFD) theory [1-3], a simple, physically-intuitive approach whose cornerstone is the idea that equations of state for chainlike molecules can be assembled by judiciously combining the equations of state for the segments and groups of segments along the chain. The derivation of the Generalized Flory-Dimer theory [2] and its predecessor, the Generalized Flory theory [1], requires the introduction of two key concepts: the insertion probability and the osmotic equation of state. The insertion probability is the probability of inserting a single test chain into a chain fluid at a given volume fraction (in thermodynamic terms, it is closely related to the fugacity). The osmotic equation of state is an exact equation which gives the pressure in terms of the insertion probability. The procedure in the development of the GF and GFD theories is to estimate the chain insertion probability in terms of monomer and dimer insertion probabilities and then to obtain the chain equation of state by substituting the chain insertion probability into the osmotic equation of state. Since the monomer and dimer insertion probabilities can be obtained from monomer and dimer equations of state, the GFD approach yields an expression for the chain equation of state in terms of the monomer and dimer equations of state.

In this progress report we summarize work accomplished under DOE sponsorship for the period December 1993 to December 1996. In section 2, we summarize the stated objectives of our previous (1993) proposal, indicating which work has been accomplished, which work is continu- 
ing, and which work has been discontinued. In section 3 , we summarize the three new objectives that were added after December 1993. In section 4, we provide a detailed description of the work accomplished, omitting those descriptions that appear in the accompanying proposal. In section 5 , we describe our human resource development efforts. Finally, in section 6 we list the publications resulting from this work. Abstracts of these papers are presented in the appendix.

\section{Summary of Progress Towards Original Objectives}

The stated objectives of our 1993 proposal to the Department of Energy were the following:

1. Develop a Generalized Flory Dimer theory for mixtures of homonuclear hard chains whose species contain segments of different sizes, and then extend this to include the case in which species also contain segments with different attractive interaction energies.

2. Develop a Generalized Flory Dimer theory for pure fluids and fluid mixtures containing heteronuclear hard chains, e.g. copolymers, and then extend this to include heteronuclear attractions.

3. Develop a Generalized Flory Dimer theory for pure fluids and fluid mixtures containing hard chains of overlapping spheres and then extend this to include constraints on bond angles, as well as attractions.

4. Perform molecular dynamics simulations to determine the transport coefficients of short hardchain and square-well chain fluids.

5. Attempt to correlate the resulting transport coefficients with estimates of the free volume based on the Generalized Flory Dimer theory.

6. Attempt to develop a kinetic theory of the dense-gas Enskog type for hard dimers and, if successful, extend this to short chain molecules.

Our progress towards these objectives is summarized below.

1. Develop a GFD theory for homonuclear mixtures.

We completed the development of a GFD theory for binary mixtures composed of hard chains of variable length and segment diameter. This work is described in detail in the attached proposal. We are still working on incorporating the effects of different segmental attractions; this is one of the objectives of the proposed research.

2. Developing a GFD theory for heteronuclear chain fluids.

We completed the development of a GFD theory for heteronuclear hard-chain fluids. This work is described in the attached proposal. We are still working on incorporating the effects of heteronuclear attractions; this is one of the objectives of the proposed research. 
3. Develop a GFD theory for fluids containing chains of overlapping spheres.

We completed the development of a GFD theory for fused hard-chain fluids with bond-lengthto-bead-diameter ratio $\approx 0.4$. We also performed Monte Carlo simulations to verify the results. This work is described in detail in Section 4. We decided not to extend this work to include the effects of bond-angle constraints or attractions because simpler models, such as the square-well chain model, appear to be adequate to the task of correlating the experimentally observed properties of real fluids.

4. Molecular Dynamics Simulations of Transport Coefficients

We completed our molecular dynamics study of the transport coefficients of short hard-chain molecules. This work is described in detail in Section 4. We decided not to extend this work to square-well chain fluids, preferring instead to investigate the dynamical properties of hard chain fluids containing much longer chains.

Objectives 5 and 6 were not attempted. Instead we decided to pursue new lines of inquiry that appeared to be more promising and that were more suited to our strengths and capabilities. These are described below.

\section{New Objectives}

In addition to the original proposed objectives summarized above, we added three new objectives to the project. These were:

1. to perform equilibrium molecular dynamics simulations on systems of very long hard-chain molecules in an effort to learn how entanglements affect the dynamic properties of polymer melts.

2. to perform molecular dynamics simulations of the static and dynamic properties of hard-chain polymer networks

3. to study the permeation of gaseous penetrants in polymer membranes.

Detailed descriptions of the work associated with the first two objectives are provided in the attached proposal. A detailed description of the work associated with the third objective is provided in Section 4. 


\section{Technical Description of Work Accomplished}

\subsection{Transport Coefficients for Fluids Containing Short Hard Chains}

In an effort to stimulate attempts to develop theories that describe the transport properties of chain fluids, we have performed discontinuous molecular dynamics simulations to calculate the self diffusion coefficient, shear and longitudinal viscosities, and thermal conductivities of fluids containing hard chains of lengths $n=1,2,4,8$, and 16 , at volume fractions $\eta$ ranging from 0.1 to 0.5 [4]. This is the most extensive study to date of the transport coefficients of short chain-like molecules. Transport coefficients were calculated by using the mean-squared displacement algorithm of Alder [5]. In this algorithm, we monitor the mean-squared displacements of position (to calculate the self diffusion coefficients), of momentum (to calculate viscosity), and of energy (to calculate the thermal conductivity). The results are displayed in Figure 1, which also shows the Enskog theory result for hard-sphere properties for comparison purposes. As expected, the diffusion coefficient decreases with both chain length and volume fraction; the shear and longitudinal viscosity increase with both chain length and volume fraction, and the thermal conductivity increases with volume fraction but decreases with chain length.

\subsection{Monte Carlo Simulation and GFD Theory for Fused-Hard-Sphere Chain Fluids}

In an effort to incorporate more realistic features into our molecular models, we have considered the fused hard sphere (FHS) chain model, in which adjacent hard spheres along the chain overlap [6]. The bond-length-to-bead diameter ratio is equal to 0.4 , the same as in polyethylene. Monte Carlo simulations were performed on FHS chain fluids containing chains of length $n=4,8$, and 16 at volume fractions ranging from 0.05 to 0.45 . The GFD theory was extended to the description of these fluids. This was not as straightforward as might be expected because identifying the underlying monomer and dimer fluids for FHS chain fluids is not obvious. In the GFD theory for tangent hard chains, the underlying monomer and dimer fluids that are created by breaking every bond or every other bond along the chain have the same volume fraction as the chain fluid, but for FHS chain fluids, the monomer and dimer fluids created in this way have a much higher volume fraction than the original FHS chain fluid. We introduced several different GF and GFD fluids, each of which has a different set of underlying monomer and dimer fluids. The most successful of these in comparison to simulation is the GFD-AC theory in which the bond-length-to-bead-diameter ratio of the underlying dimer fluid is taken to be identical to that of the FHS chain fluid, but the diameter and number of beads per unit volume are adjusted so that the surface area and volume fraction are the same as for the FHS chain fluid. 

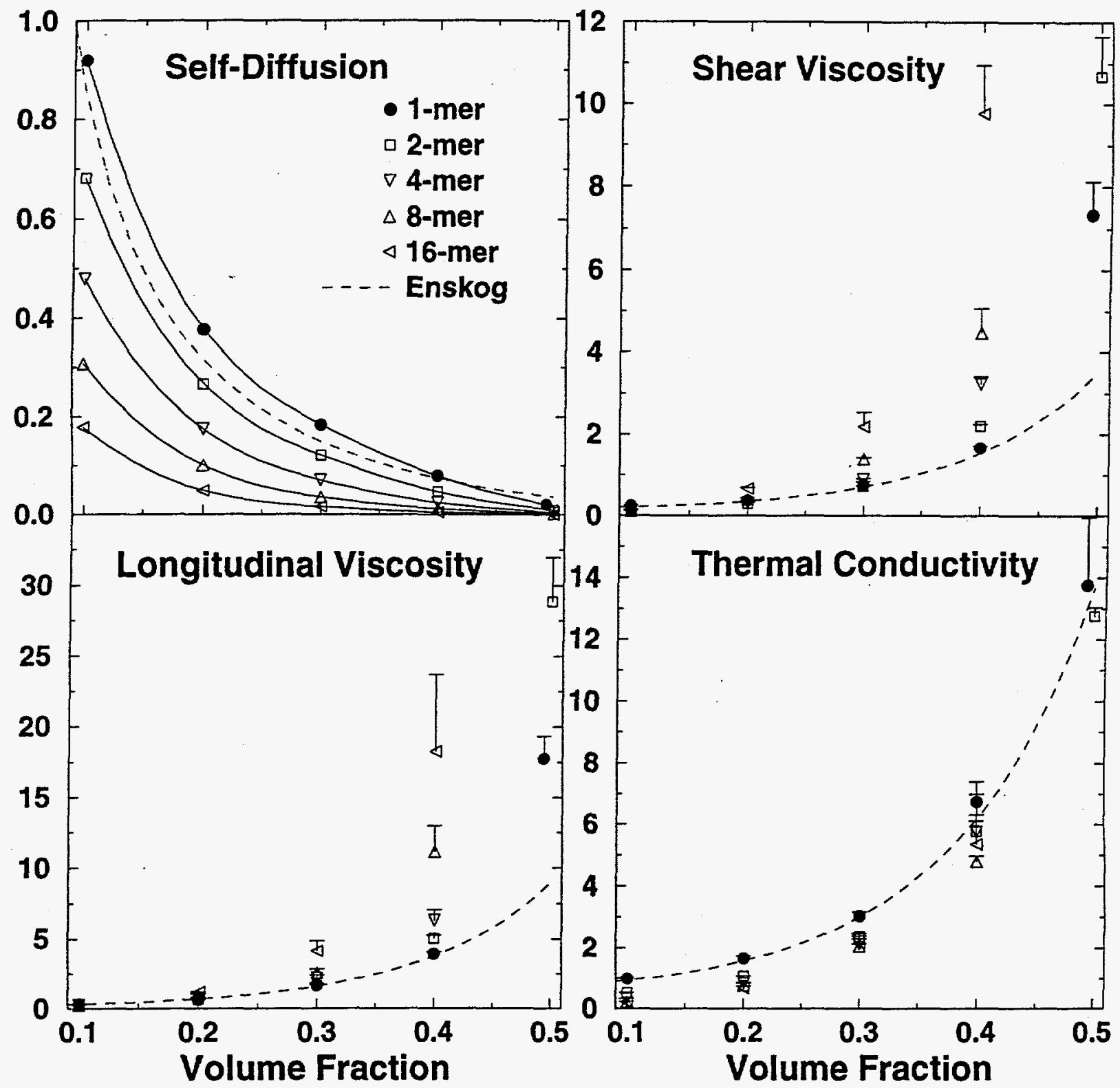

Figure 1: Reduced transport coefficients for fluids containing chains of lengths $n=2,4,8,16$ [4]: 


\subsection{Permeation of Penetrants Through Polymer Membranes}

In 1994, we began a program of research aimed at modeling the permeation of penetrants through polymer membranes, with special focus on the phenomenon known as facilitated transport. In facilitated transport, specific interaction sites (typically metal salts) chosen for their affinity to one of the components of a gas mixture that needs to be separated, are covalently attached to the chains in the membrane. The presence of facilitating sites dramatically alters the solubility of this component and, since penetrant permeability is the product of penetrant diffusivity and solubility, can lead to enhanced permeability and hence enhanced selectivity for the component of interest.

In an effort to learn more about the diffusion and solubility of gaseous penetrants in polymer membranes, we conducted a series of three computer simulation studies. These are described below.

\subsubsection{Grand Canonical Molecular Dynamics Simulations of the Diffusion of Hard Sphere Penetrants in Stationary Hard Sphere and Hard Chain Membranes}

In the first study [7], Grand Canonical Molecular Dynamics (GCMD) was used to simulate the diffusion of hard-sphere penetrants in stationary membranes modeled in two ways : as a collection of hard spheres, and as a collection of hard chains. In the GCMD technique, which was introduced by Papadopoulou et al. [8] and embellished by Heffelfinger and van Swol [9], molecular dynamics is performed in a simulation cell which is maintained at a constant chemical potential gradient by performing grand canonical simulations in control volumes which flank the main simulation cell. The penetrant mutual diffusivity is calculated using Fick's law which relates the penetrant flux to the concentration gradient.

Highlights of our results are the following. Penetrant mutual diffusivity is found to be a function of two competing factors, a thermodynamic factor and a friction factor. An increase in the chemical potential of the entering penetrant tends to increase the diffusivity (the thermodynamic factor effect), while a corresponding increase in the number of penetrants within the system tends to decrease the diffusivity (the friction factor effect). When the penetrant beads are of the same size or larger than the polymer segments, diffusivity is dominated by the thermodynamic factor, which means that increasing the chemical potential leads to an increase in the diffusivity. When the penetrant beads are smaller than the polymer segments, diffusivity is dominated by the friction factor, which means that an increase in the chemical potential leads to a decrease in the diffusivity. As expected the mutual diffusivity decreases with increasing packing fraction and increases with increasing chain length of the stationary barrier media. Penetrant tracer diffusivity in the stationary barrier media is calculated using standard molecular dynamics simulation and is found to be almost an order of magnitude smaller than penetrant mutual diffusivity. This suggests that the Darken equation [10], which equates the two and is often used as a justification for comparing simulation values of the tracer diffusivity with experimental values of the mutual diffusivity [11], is incorrect. 


\subsubsection{Grand Canonical Monte Carlo Simulations of the Sorption of Penetrants in Barrier Media}

In the second study [12], Grand Canonical Monte Carlo simulations of penetrant solubility in polymer membranes have been used to predict sorption isotherms as a function of polymer-penetrant interactions and temperature. The polymer is modeled as a collection of hard chains, a collection of square-well chains, and a collection of facilitating chains (square-well chains with specific "facilitating" sites that exhibit a special affinity for the penetrants). The penetrants are modeled as hard-spheres and as square-well spheres. Partition coefficients have been compared for stationary and moving polymers, with the stationary polymeric media modeling glassy polymers and the moving polymeric media modeling rubbery polymers. Partition coefficients are higher in the moving barrier media than in the stationary barrier media. Partitioning was also studied as a function of the concentration and relative strength of facilitating sites. Partition coefficients increased with increasing concentration of facilitating sites and with increasing strength of the attraction between facilitating sites and penetrants. The presence of a second penetrant species of normal affinity is found to decrease the partitioning of the penetrant with special affinity for facilitating sites.

\subsubsection{Grand Canonical Molecular Dynamics Simulation of the Diffusion of Square-Well Pen- etrants in Square-Well Chain Membranes}

In the third study [13], Grand Canonical Molecular Dynamics is used to calculate the mutual diffusivity of penetrants in polymer membranes. The polymer is modeled as a collection of both stationary and moving square-well chains, and as a collection of both stationary and moving facilitating chains, while the penetrants are modeled as square-well spheres. By combining the mutual diffusivity results with the penetrant partitioning and solubility results of the study described above, we can calculate the penetrant permeability as a function of reservoir chemical potential, barrier packing fraction, and barrier chain length for a variety of penetrant/polymer combinations. Penetrant diffusivity decreases with increasing barrier packing fraction and with increasing attraction between penetrant and polymer (due to the lower penetrant mobility). Penetrant diffusivity is found to be larger when the barrier is moving than when the barrier is stationary. Penetrant permeability (which is the product of the diffusivity and solubility) decreases with increasing concentration and strength of facilitating sites in the barrier, in contrast to some experimental findings. Although the trends in penetrant diffusivity and solubility are identical to those observed experimentally, (solubility increases and diffusivity decreases with increasing concentration of facilitating sites), the increase in solubility is not enough to offset the decrease in diffusivity. We are now trying to determine the conditions at which permeability will be enhanced by the addition of facilitating sites. 


\subsection{Computer Simulation Study of the "Collapse" of a Single Square-Well Chain}

Off-lattice Monte Carlo and discontinuous molecular dynamics simulations were performed on a homopolymer molecule modeled as a freely-jointed chain of square-well spheres $[14,15]$. The equilibrium mean-square radius of gyration, internal energy, and heat capacity were obtained for isolated square-well chains of length $n=3-8,12,16,32$, and 64 as a function of reduced temperature. Much to our surprise, we found that in addition to exhibiting the well-known second-order coil-to-globule collapse transition, our 64-mer chain exhibited two additional transitions at lower temperatures: a first-order liquid-solid transition and a second-order solid-solid polymorphic transition.

The analogies between the types of states found for this homopolymer chain and the types of states found for a heteropolymer chain, e.g. a protein molecule, are striking. Our analysis shows that a homopolymer chain can exist in three or more states: a random coil state (gas-like phase), a disordered compact chain (a liquid-like phase), and one or more ordered compact chain states (solid-like phases). In comparison, linear peptide chain molecules containing various amino acid side chains have been found experimentally to exist in a denatured state, a molten globule state, and a unique native state [16]. The "gas" to "liquid-like" transition in the homopolymer corresponds to the hydrophobic collapse to a disordered globule found in at least some proteins [17], and observed as the first step in some lattice simulations of protein folding [18]. The liquid-to-solid transition in the homopolymer can be identified with the collapsed globule to native state transition in proteins. Both are disorder-to-order transitions and both are finite-system first-order-like transitions in the sense that two-states are involved with a low probability in between. With this identification, the major difference between a protein and the homopolymer studied here would be that the former has a solid-like ground state that is unique due to heterogeneity introduced by the amino acid side chains, while the later has a multiplicity of essentially equivalent solid-like states and exhibits a polymorphism which is very unusual in proteins [19], if it exists at all.

\subsection{Simulation of Double-Tethered Chains and Their Mixtures at an Impen- etrable Interface}

We have investigated the structural, conformational and dynamic properties of grafted monolayers comprised of double-tethered or "looped" chain molecules using discontinuous molecular dynamics simulation. The structural and conformational properties obtained from our continuous-space molecular dynamics compare favorably with the results of previous on-lattice bond-fluctuation simulations. Equilibrium static property scaling relationships for polymer loops are found to agree with those for polymer brushes (single-tethered chains) of half chain length in the intermediate density (or scaling) regime. We have also examined the effect of annealing on structure by allowing the anchor sites to diffuse laterally along the surface. The conformational properties of mixtures of polymers loops and brushes was also studied using the bond-fluctuation method. 
We have also studied the dynamic behavior of annealed looped chains. The relaxation time and diffusivity of annealed chains was examined as a function of anchor density and chain length. Comparing the dynamic properties of looped chains to those of polymer brushes has yielded interesting insights into the role of the second tethered site. Despite the presence of the second anchor, the scaling of the relaxation time and lateral diffusivity with chain length is similar for annealed polymer loops and polymer brushes. In contrast, the scaling of these quantities with anchor density is much stronger for annealed polymer loops than for polymer brushes.

\subsection{Other Work}

We have also investigated: (1) second virial coefficients for square-well chain fluids, (2) extensions of the Generalised Flory and thermodynamic perturbation theories using tetramer reference fluids, (3) effective pair potentials for isolated hard- and square-well chains based on Monte Carlo simulations, (4) isolated sticky chains, (5) the accuracy of the approximations used in the GFD theory, (6) gas permeation models for membrane modules, and (7) the effect of pressure on the relaxation dynamics of polypropylene oxide. Abstracts associated with these investigations are provided in the appendix.

\section{Human Resource Development}

This DOE grant was used to support the thesis research of five Ph.D students: Steven Smith, John Wichert, Suresh Sunderrajan, Harpreet Gulati, and Nirupama Ramamurthy-Kenkare. Dr. Smith is now at Dupont, (soon-to-be) Dr. Sunderrajan is at Union Camp and Dr. Wichert is seeking employment. Mr. Gulati and Ms. Ramamurthy-Kenkare should receive their Ph.D.s within a year. This DOE grant also provided partial support for the thesis research of M.S. student James Dautenhahn, now employed at Gold Line refining, and for Ph.D student, Lorenza Costa, a visiting scholar from the University of Milan. It also supported the postdoctoral training of Dr. Yaoqi Zhou, now a research associate in Harvard University's Chemistry Department.

\section{Papers Describing Work Supported by this Contract (January 1994 - December 1996)}

1. C. P. Bokis, M. D. Donohue and C. K. Hall, "Application of a Modified Generalized Flory Dimer Theory to Normal Alkanes", Ind. Eng. Chem. Res. 33, 1290 (1994).

2. C.P. Bokis, M. D. Donohue and C. K. Hall, "Second Virial Coefficients for Chain Molecules", Ind. Eng. Chem. Res. 33, 146 (1994). 
3. J. M. Wichert and C. K. Hall, "Second Virial Coefficient Calculations for Square-Well Chain Molecules", Macromolecules, 27, 2144 (1994).

4. J. M. Wichert and C. K. Hall, "Generalized Flory Equation of State for Chain - Monomer Mixtures of Unequal Segment Sizes", Chem. Eng. Sci., 17, 2793, (1994).

5. J. Dautenhahn and C. K. Hall, "Monte Carlo Simulation of Off-Lattice Polymer Chain : Effective Pair Potentials in Dilute Solution”, Macromolecules, 27, 5399 (1994).

6. L.A. Costa, Y. Zhou, C.K. Hall and S. Carra, "Fused Hard-Sphere Chain Molecules: Comparison Between Monte Carlo Simulation for the Bulk Pressure and Generalized Flory Theories", J. Chem. Phys. 102, 6212 (1995).

7. S.W. Smith, C.K. Hall and B.D. Freeman, "Large-Scale Molecular Dynamics Study of Entangled Hard-Chain Fluids", Phys. Rev. Letters, 75, 1316 (1995).

8. Y. Zhou, C.K. Hall and G. Stell, "Thermodynamic Perturbation Theory for Fused Hard-Sphere and Hard-Disk Chain Fluids", J. Chem. Phys. 103, 2688 (1995).

9. Y. Zhou, S.W. Smith and C.K. Hall, "Linear dependence on Chain Length for the Thermodynamic Properties of Tangent Hard-Sphere Chains", Molecul. Phyś., 으, 1157 (1995).

10. Y. Zhou and C.K. Hall, "Exact Results for Isolated Sticky Chains", Molecul. Phys., 6, 1485 (1995).

11. Y. Zhou and C.K. Hall, "Solute Excluded Volume Effects on the Stability of Globular Proteins: A Statistical Thermodynamic Theory", Biopolymers, $\underline{38}, 273$ (1996).

12. S.W. Smith, C.K. Hall and B.D. Freeman, "Molecular Dynamics Study of Entangled HardChain Fluids", J. Chem. Phys. 104, 5616 (1996).

13. H.S. Gulati, J.M. Wichert and C.K. Hall, "Generalized Flory Equations of State for Hard Heteronuclear Chain Molecules", J. Chem. Phys. 104, 5220 (1996).

14. S.K. Kumar, I. Szleifer, C.K. Hall and J.M. Wichert, "Computer Simulation Study of the Approximations Associated with Generalized Flory Theories", J. Chem. Phys. 104, 9100 (1996).

15. S.W. Smith, C.K. Hall and B.D. Freeman, "Smith, Hall and Freeman Reply", Phys. Rev. Letters, 76, 4449 (1996).

16. S.W. Smith, B.D. Freeman and C.K. Hall, "Corrections for Analytical Gas Permeation Models for Binary Gas Mixture Separations Using Membrane Modules", J. Membrane Sci. 118 , 289 (1996).

17. S. Sunderrajan, C.K. Hall and B.D. Freeman, "Estimation of Mutual Diffusion Coefficients in Polymer/Penetrant Systems Using Nonequilibrium Molecular Dynamics Simulations", J. Chem. Phys. 105, 1621 (1996). 
18. J.M. Wichert, H.S. Gulati and C.K. Hall, "Binary Hard Chain Mixtures. I. Generalized Flory Equations of State", J. Chem. Phys. 105, 7669 (1996).

19. H.S. Gulati, C.K. Hall, R.L. Jones and R.J. Spontak, "Equilibrium Conformations and Dynamic Relaxation of Double-Tethered Chain Molecules at an Impenetrable Interface", J. Chem. Phys. 105, 7712 (1996).

20. Y. Zhou, C.K. Hall and M. Karplus, "A First-Order Disorder-to-Order Transition in an Isolated Homopolymer Model”, Phys. Rev. Letters, 77, 2822 (1996).

21. S.W. Smith, C.K. Hall and B.D. Freeman, "Discontinuous Molecular Dynamics of Polymeric Fluids", J. Computational Phys. (in press).

22. S.W. Smith, B.D. Freeman and C.K. Hall, "Pressure-Dependent Photon Correlation Spectroscopic Investigation of Poly(Propylene Oxide) Near the Glass Transition", Macromolecules (in press).

23. H. S. Gulati, D. C. Driscoll, R. J. Spontak and C. K. Hall, "Conformational and Dynamic Properties of Polymer Loops and Their Mixtures at an Impenetrable Interface", Proc. of Mat. Res. Soc., Boston (1996), (in press).

24. S. Sunderrajan, C.K. Hall and B.D. Freeman, "Sorption Isotherms for Spherical Penetrants in Facilitating Polymeric Media using Monte Carlo Simulations", Mol. Phys. (submitted).

25. S. Sunderrajan, C.K. Hall and B.D. Freeman, "Chemical Potential Gradient Driven Permeation of Small Molecules Through Polymeric Media", J. Chem. Phys. (submitted).

\title{
Appendix: Abstracts
}

\section{Papers Published}

1. "Application of a Modified Generalized Flory Dimer Theory to Normal Alkanes", C. P. Bokis, M. D. Donohue and C. K. Hall, Ind. Eng. Chem. Res., 33,1290 (1994).

\begin{abstract}
The applicability of chain equations of state to real systems is discussed in this paper. For this purpose, we have compared four theories: the Perturbed-Hard-Chain theory (PHTC) of Prausnitz and co-workers, the Generalized Flory (GF) and Generalized Flory Dimer (GFD) theories of Hall and co-workers, and the Statistical Associating Fluid theory (SAFT) of Radosz, Gubbins and co-workers. In our comparison, the perturbation expansion in the attractive term was truncated after the first order term for all theories. Comparison of these theories with Monte Carlo simulation data for hard chains and square-well chains showed that the GFD theory, which explicitly takes into account the effect of the
\end{abstract}


formation of chains in both the repulsive and attractive part of the equation, is in best agreement with the data. The GFD theory was further improved by using the simulation data directly to reevaluate the shape parameters ' $c$ ' and ' $q$ ', which were found to be density dependent. The new simplified GFD theory gives a significantly better correlation of the properties of normal alkanes than the other four theories.

2. "Second Virial Coefficients for Chain Molecules", C. P. Bokis, M. D. Donohue and C. K. Hall, Ind. Eng. Chem. Res., 33, 146 (1994).

\begin{abstract}
The importance of having accurate second virial coefficients in phase equilibrium calculations, especially for the calculation of dew points, is discussed in this paper. The squarewell potential results in a simple but inaccurate equation for the second virial coefficient for small, spherical molecules such as argon. Here, we present a new equation for the second virial coefficient of both spherical molecules and chain molecules which is written in a form similar to that for the square-well potential. This new equation is accurate in comparison to Monte Carlo simulation data on second virial coefficients for square-well chain molecules and with second virial coefficients obtained from experiments on $n$-alkanes.
\end{abstract}

3. "Second Virial Coefficient Calculations for Square-Well Chain Molecules", J. M. Wichert and C. K. Hall, Macromolecules 27,2144 (1994).

\begin{abstract}
The second virial coefficient, $B_{2}$, has been calculated for square-well chain molecules of lengths $n=2$ to 50 and well widths of $\lambda=0.25$ to 1.0 by Monte Carlo integration. The theta temperature, at which $B_{2}=0$, is independent of chain length for $\lambda \approx 0.5$; increases with chain length for $\lambda>0.5$, and decreases with chain length for $\lambda<0.5$. A scaling relation, $T_{\theta}^{*}(n)-T_{\theta}^{*}(\infty) \propto n^{-\phi}$, accurately describes the departure of the theta temperature from the infinite length value for $\lambda \geq 0.6$. A closed form expression for the second virial coefficient of square-well chains is presented which accurately fits the Monte Carlo data for $n=2-50$ and $\lambda=0.25-0.75$. When compared to Monte Carlo results, the second virial coefficient predicted by the generalized Flory-dimer theory for square-well chains is found to be increasingly inaccurate as chain length increases. If we correct the generalized Flory-dimer equation of state by forcing it to have the second virial coefficient, the compressibility factor is accurately predicted at densities below $\eta=0.05$.
\end{abstract}

4. "Generalized Flory Equation of State for Chain - Monomer Mixtures of Unequal Segment Sizes", J. M. Wichert and C. K. Hall, Chem. Eng. Sci. 17, 2793, (1994).

Abstract 
A Generalized Flory equation of state is derived for chain/monomer mixtures, where the monomer diameters are different than the chain segment diameters. An alternate derivation of the osmotic equation of state for binary mixtures is also presented; the equation is derived by inserting both species simultaneously into the fluid. The alternate equation of state predicts the conformal solution mixing rule when the monomer and chain segments are the same diameter. Chain insertion probabilities, required in the osmotic equation of state in order to predict thermodynamic properties, are estimated using monomer mixture insertion probabilities based on the Mansoori, Carnahan, Starling and Leland equation of state and the Generalized Flory theory. An extensive set of 4-mer/monomer and 8mer/monomer Monte Carlo simulation have also been performed. Simulation compressibility factors are compared to the new equation of state's predictions; agreement is quite good.

5. "Monte Carlo Simulation of Off-Lattice Polymer Chain : Effective Pair Potentials in Dilute Solution", J. Dautenhahn and C. K. Hall, Macromolecules, 27, 5399 (1994).

\begin{abstract}
The potential of mean force, $U(r)$, between two polymers is calculated using Monte Carlo methods. Two models of polymers as off-lattice chains of tangent hard spheres are used. In the first, the beads of the chains are simply hard spheres (the athermal case). In the second, the beads of the chains are allowed to interact with other non-adjacent beads on the same chain or with beads on a different chain via a square-well potential. For both models, $U(r)$ is shown to decrease as chain length increases. This is in contrast to the FloryKrigbaum theory but is in agreement with previous findings of Grosberg et al. . For chains of different lengths, $U(r)$ decreases as the difference between the lengths of chains increases. Near the $\theta$-point,the $U(r)$ curves tend to be indistinguishable for all chain lengths. Scaling analysis of the second virial coefficient for athermal chains gives a scaling exponent of $\gamma=0.272 \pm 0.005$, in agreement with the findings of Yethiraj et al. . From analysis of both the second virial coefficient and the radius of gyration of single chains, the $\theta$-point is found to be at a square-well potential of $\epsilon_{\theta}=-0.32 k T$, in agreement with the results of Wichert and Hall. A correlation for $U(r)$ that fits the simulation data reasonably well is also presented.
\end{abstract}

6. L.A. Costa, Y. Zhou, C.K. Hall and S. Carra, "Fused Hard-Sphere Chain Molecules: Comparison Between Monte Carlo Simulation for the Bulk Pressure and Generalized Flory Theories", J. Chem. Phys., 102, 6212 (1995).

\begin{abstract}
We report Monte Carlo simulation results for the bulk pressure of fused-hard-sphere (FHS) chain fluids with bond-length-to-bead-diameter ratios $\approx 0.4$ at chain lengths $n=4$, 8 and 16. We also report density profiles for FHS chain fluids at a hard wall. The results for the compressibility factor are compared to results from extensions of the Generalized Flory (GF) and Generalized Flory Dimer (GFD) theories proposed by Yethiraj et. al. and
\end{abstract}


by us. Our new GF theory, GF-AB, significantly improves the prediction of the bulk pressure of fused-hard-sphere chains over the GFD theories proposed by Yethiraj et. al. and by us although the GFD theories give slightly better low-density results. The GFD-A theory due to Yethiraj et. al. and the new theories (GF-AB, GFD-AB, and GFD-AC) satisfy the exact zero-bonding-length limit. All theories considered recover the GF or GFD theories at the tangent hard-sphere chain limit.

7. S.W. Smith, C.K. Hall and B.D. Freeman, "Large-Scale Molecular Dynamics Study of Entangled Hard-Chain Fluids", Phys. Rev. Letters, 75, 1316 (1995).

\begin{abstract}
Equilibrium molecular dynamics is used to simulate fluids comprised of chains of tangent hard spheres. Reptation theory predictions of segmental motion are compared with simulation results. In addition to the usual tube confinement, a second entanglement effect is observed. As the chain disengages from the tube, persistent interchain contacts cause a plateau in the segment mean-square displacement (MSD) and subsequent accelerated diffusion. Associated with the plateau in the MSD is a corresponding delay in relaxation of the end-to-end vector as interior chain segments are extended during disentanglement.
\end{abstract}

8. Y.Zhou, C.K. Hall and G. Stell, "Thermodynamic Perturbation Theory for Fused Hard-Sphere and Hard-Disk Chain Fluids", J. Chem. Phys. 103, 2688 (1995).

\begin{abstract}
We find that first-order thermodynamic perturbation theory (TPT1) which incorporates the reference monomer fluid used in the generalized Flory-AB (GF-AB) theory yields an equation of state for fused hard-sphere (FHS) chain fluids that has accuracy comparable to the GF-AB and GF-dimer-AC theories. The new TPT1 equation of state is significantly more accurate than other extensions of the TPT1 theory to FHS chain fluids. The TPT1 is also extended to two-dimensional fused hard-disk chain fluids. For the fused hard-disk dimer fluid, the extended TPT1 equation of state is found to be more accurate than the Boublik hard-disk dimer equation of state.
\end{abstract}

9. Y. Zhou, S.W. Smith and C.K. Hall, "Linear dependence on Chain Length for the Thermodynamic Properties of Tangent Hard-Sphere Chains", Molecul. Phys., 86, 1157 (1995).

\begin{abstract}
Equilibrium molecular dynamics simulation techniques are used to obtain accurate compressibility factors ( $\leq 0.1 \%$ error) for tangent hard-sphere (THS) chains of lengths 2 $8,16,32,64,96$, and 192 . Our simulation results show that within simulation statistical errors, the dependence of compressibility factors on chain length approaches linearity very rapidly. At volume fractions of 0.4 or above, the linearity starts at a chain length of 3 while at volume fractions of 0.1 or above, the linearity starts at a chain length of 6 . The linearity
\end{abstract}


seems to be exactly satisfied. The thermodynamic perturbation theory (TPT) and the generalized Flory (GF) theory equations of state are extended to become a linear combination of the compressibility factors of any two reference THS chain fluids. It is found that the extended GF theory is identical to the extended TPT (ETPT) when the excluded volumes of chains and reference chains are assumed to be linearly dependent on the chain length. Our simulation data implies that using reference chain fluids containing chain lengths of 3 or more provides a near exact THS-chain equation of state at volume fractions $\eta>0.4$. For $\eta>0.2$, reference chain fluids of chain length 4 or more are required and for $\eta>0.1$, reference chain fluids of chain length 6 or more are required. As an illustrative example, a new equation of state for the THS chain fluids is obtained from the linear combination of 4-mer and 8-mer equations of state. The new equation of state remarkably reproduces the simulation data on 192-mers at $\eta>0.2$. For the low volume fraction of 0.1 , its relative error is only $3 \%$.

10. Y. Zhou and C.K. Hall, "Exact Results for Isolated Sticky Chains", Molecul. Phys., 86, 1485 (1995).

\begin{abstract}
Exact results for the thermodynamic properties of isolated freely-jointed sticky trimers and tetramers are obtained by taking appropriate limits on the exact results for a single square-well chain. Two new methods for approximating the square-well potential by a sticky potential are suggested. These new methods greatly extend the range of squarewell widths over which the thermodynamic properties of a single square-well chain can be accurately estimated in terms of the corresponding properties of a single sticky chain.
\end{abstract}

11. Y. Zhou and C.K. Hall, "Solute Excluded Volume Effects on the Stability of Globular Proteins: A Statistical Thermodynamic Theory", Biopolymers, 38, 273 (1996).

\begin{abstract}
A statistical thermodynamic theory is developed to investigate the effects of solute excluded-volume on the stability of globular proteins. Proteins are modeled as two states in chemical equilibrium: the denatured state is modeled as a flexible chain of tangent hard spheres (pearl-necklace chain) while the native state is modeled as a single hard sphere. Study of model proteins BPTI and lysozyme in a McMillan-Mayer model solution of hardspheres indicates that the excluded volume of solutes has three distinct types of effects on protein stability: 1) small-size solutes strongly denature proteins, 2) medium-size solutes stabilize proteins at low solute concentrations and destabilize them at high concentrations, and 3) large-size solutes stabilize native-state proteins across the whole liquid region. The study also finds that increasing the chain length of hard-chain polymer solutes has an effect on protein stability that is similar to increasing the diameter of spherical solutes. This work qualitatively explains why stabilizers tend to be large size molecules such as sugars, polymers, polynols, nonionic and anionic surfactants while denaturants tend to be small size molecules such as alcohols, glycols, amides, formamides, ureas, and guanidium
\end{abstract}


salts. Quantitative comparison between theoretical predictions and experimental results for folding free energy changes shows that the excluded-volume effect is at least as important as the binding and/or electrostatic effects on solute-assisted protein-denaturation processes. Our theory may also be able to explain the effect of excluded volume on the $\Phi$-condensation of DNA.

12. S.W. Smith, C.K. Hall and B.D. Freeman, "Molecular Dynamics Study of Entangled HardChain Fluids", J. Chem. Phys. 104, 5616 (1996).

\begin{abstract}
By applying efficient computational algorithms to the simplest off-lattice polymer model - the freely-jointed tangent hard-sphere chain - we have been able to perform molecular dynamics simulations long enough to probe chain dynamics in the entangled regime. Chain lengths range from 8 to 192 segments while volume fractions range from 0.3 to 0.45. Analysis of the mean-square displacement (MSD), Rouse modes, scattering functions, and end-to-end vector correlations provides information about chain motion. Chain dynamics are compared with predictions of the Rouse model for short chains and the tube model of Doi and Edwards for long chains. The mean-square displacement for the inner segments of the longest chains are consistent with predictions of the tube model, reproducing the three scaling regimes that are postulated to occur. In addition, anomalous diffusive behavior in the atomic MSD of the inner segments is observed at long times as the inner segments cross over into the free diffusion limit. Rouse-mode autocorrelation functions decay non-exponentially and do not exhibit scaling consistent with the tube model. Definitive plateau-like behaviors are observed in the density-density correlations, normal coordinate decay, and end-to-end vector relaxation of the 192-mer fluids at the highest density.
\end{abstract}

13. H.S. Gulati, J.M. Wichert and C.K. Hall, "Generalized Flory Equations of State for Hard Heteronuclear Chain Molecules”, J. Chem. Phys. 104, 5220 (1996).

\begin{abstract}
The Generalized Flory (GF) and Generalized Flory-Dimer (GFD) equations of state have been extended to fluids containing hard heteronuclear chain-like molecules. Compressibility factor expressions have been derived for block, alternating and random "copolymer" fluids. The effect of composition and the relative size of the segments of a heteronuclear chain on the compressibility factor are studied. We have also performed molecular dynamics computer simulations on these systems using an extension of Rapaport's algorithm in which the chains are effectively treated as hard spheres of different sizes held together by invisible strings. The compressibility factors predicted by the GFD theory for heteronuclear chain fluids are in very good agreement with our computer simulation results. The predictions of Chiew's Percus Yevick theory are also in very good agreement with our computer simulation results on block copolymers.
\end{abstract}


14. S.K. Kumar, I. Szleifer, C.K. Hall and J.M. Wichert, "Computer Simulation Study of the Approximations Associated with Generalized Flory Theories", J. Chem. Phys. 104, 9100 (1996).

\begin{abstract}
The Chain Increment Method and Configurational Bias Monte Carlo methods are used to test the approximations made in the derivation of the Generalized Flory-Dimer (GF-D) theory for tangent hard sphere chains. Insertion probabilities and residual chemical potentials are calculated for hard chain fluids containing chains of length $n=4,8,16$ and 32 at monomer densities, $\rho_{M}$, up to 0.8 . We find that the largest errors in the GF-D theory are those associated with assuming that the probability of inserting a monomer into a chain fluid is approximately equal to the probability of inserting a monomer into a monomer fluid, as predicted by the Carnahan-Starling equation of state. The errors in the incremental compressibility factor of the second segment associated with assuming that the conditional probability of inserting a second bead next to the first bead in a chain fluid is approximately equal to the probability of inserting a second bead next to the first bead in a dimer fluid as predicted by combining the Camahan-Starling and Tildesley-Streett equations of state is relatively small. Consistent with the findings of Mooij and Frenkel, we find that these two approximations lead to an overprediction of the incremental contributions to the compressibility factor. Despite the overprediction of the incremental contributions to the compressibility factor of the first segment, the GF-D equation of state accurately predicts the compressibility of hard chains; this accuracy is traced to (1) the insensitivity of the compressibility factor to errors in the insertion probability and (2) cancelation of errors in the incremental compressibility factor of the first segment with small cumulative errors in the incremental compressibility factors of the third and subsequent segments.
\end{abstract}

15. S.W. Smith, B.D. Freeman and C.K. Hall, "Corrections for Analytical Gas Permeation Models for Binary Gas Mixture Separations Using Membrane Modules", J. Membrane Sci. 118, 289 (1996).

\begin{abstract}
Corrected solutions for analytical models, originally presented by Rautenbach and Dahm, for membrane-based binary gas separation are presented. These models are based on constant pressure in the feed and permeate streams and perfect-mixing in the permeate stream. A comparison of these models with more complete numerical solutions suggests that the analytical models provide excellent short-cut, preliminary design information. The analytical solutions may also be used to assist convergence of more complete numerical methods.
\end{abstract}

16. S. Sunderrajan, C.K. Hall and B.D. Freeman, "Estimation of Mutual Diffusion Coefficients in Polymer/Penetrant Systems Using Nonequilibrium Molecular Dynamics Simulations", J. Chem. Phys. 105, 1621 (1996).

Abstract 
Experimental studies of the diffusion of small molecules in polymers typically report mutual diffusivities while simulation studies report tracer diffusivities. The Darken equation has been used to relate measured mutual diffusivities to calculated tracer diffusivities. The current study uses Grand Canonical Molecu lar Dynamics (GCMD) to simulate a gradient in chemical potential and calculate the mutual diffusivi ty in a polymer/penetrant system. The penetrants are modeled as hard spheres while the polymer is modeled as a collection of stationary hard chains. Penetrant mutual diffusivity is a function of two competing factors, a thermodynamic factor and a friction factor. An increase in the chemical potential of the penetrant tends to increase the diffusivity, the thermodynamic factor effect, while a corresponding increase in the number of particles within the system tends to decrease the diffusivity, the friction factor effect. Mutual diffusivity differ $s$ from penetrant tracer diffusivity by almost an order of magnitude. Mutual diffusivities do not obey the Darken relation for the simulation model used in this study.

17. J.M. Wichert, H.S. Gulati and C.K. Hall, "Binary Hard Chain Mixtures. I. Generalized Flory Equations of State”, J. Chem. Phys. 105, 7669 (1996).

\begin{abstract}
In this series of two papers we study the thermodynamics of binary hard chain mixtures. Here, a generalized Flory-dimer (GF-D) equation of state is derived for binary hard chain mixtures composed of chains of variable length and segment diameter. Compressibility factors predicted by the GF-D equation of state developed here and by the previously derived generalized Flory equation of state are compared to previous Monte Carlo results for hard monomer/hard chain mixtures, and to new molecular dynamics (MD) hard monomer/hard chain and hard chain/hard chain mixture simulation results. Compared to the MD simulations, the GF-D theory is found to be quite accurate, with an average error of about $3 \%$ at liquid-like densities.
\end{abstract}

18. H.S. Gulati, C.K. Hall, R.L. Jones and R.J. Spontak, "Equilibrium Conformations and Dynamic Relaxation of Double-Tethered Chain Molecules at an Impenetrable Interface", J. Chem. Phys. 105, 7712 (1996).

\begin{abstract}
In this work, the static conformational properties and dynamic relaxation behavior of monolayers of chain molecules grafted at both ends (polymer "loops") to an impenetrable plane are investigated utilizing off-lattice discontinuous molecular dynamics (DMD) simulations. The conformational properties observed from DMD simulation are compared to the results of a previous on-lattice Bond Fluctuation (BF) study. This provides a unique opportunity to decouple the effects of excluded volume, chain flexibility and interparticle interactions, since these effects are treated differently in the two simulation methods. Static equilibrium properties of the looped chain layers determined from the DMD and BF models are in qualitative agreement with each other and with self-consistent field (SCF) predictions for brushes of $N / 2$ repeat units. By allowing the chain anchors to move laterally along the interfacial plane, the effect of annealing on the layer properties is also
\end{abstract}


investigated. The characteristic relaxation time of the annealed looped chains, discerned from radius of gyration autocorrelation functions, obeys a scaling relationship of the form $\tau \sim N^{3} \sigma^{4 / 3}$, where $\sigma$ is the surface density of the tethered chain ends. The lateral selfdiffusion coefficient of the chains during annealing is also measured, with lateral diffusivity scaling as $N^{\alpha} \sigma^{\beta}$. The exponents $\alpha$ and $\beta$ undergo a smooth transition from $\alpha \approx-4 / 3$, $\beta \approx-4 / 3$ at low densities to $\alpha \approx-2, \beta \approx-2$ at high densities. Both relaxation times and lateral diffusivity exhibit a different density scaling than brushes with $N / 2$ repeat units.

19. Y. Zhou, C.K. Hall and M. Karplus, "A First-Order Disorder-to-Order Transition in an Isolated Homopolymer Model”, Phys. Rev. Letters, 77, 2822 (1996).

\begin{abstract}
The thermodynamic behavior of an isolated freely-jointed homonuclear square-well 64 mer chain is studied by discontinuous molecular dynamics (DMD) simulations. It is shown that there are three transitions that can be described as gas-liquid, liquid-solid, and solid-solid polymorphic transitions. The liquid-solid-like transition is a first-order (twostate) disorder-to-order transition. Implications of the results for protein folding are briefly discussed.
\end{abstract}

\title{
2. Papers in Press
}

1. S.W. Smith, C.K. Hall and B.D. Freeman, "Discontinuous Molecular Dynamics of Polymeric Fluids", J. Computational Phys. (in press).

\begin{abstract}
Molecular dynamics simulation techniques for systems interacting with discontinuous potentials are discussed. Optimization and efficiency techniques are summarized for performing discontinuous molecular dynamics on serial computers with direct application to polymer-like fluids. Comparisons are presented for two algorithms: (1) single-event scheduling, and (2) multiple-event scheduling. The single-event scheduling algorithm is approximately $75 \%$ faster than the multiple-event scheduling algorithm for molecular fluids but yields equivalent performance for atomic fluids. For the single-event scheduling method, a combination of link lists and neighbor lists are used when searching for possible particle interactions. The combination of efficiency techniques permits multi-billion time step simulations for relatively large systems on desktop workstations. Both discontinuous molecular dynamics codes for single and multiple-event scheduling algorithms are available on the Internet. The utility of the method is demonstrated for entangled chains, tethered chains, and heteronuclear chain mixtures.
\end{abstract}

2. S.W. Smith, B.D. Freeman and C.K. Hall, "Pressure-Dependent Photon Correlation Spectroscopic Investigation of Poly(Propylene Oxide) Near the Glass Transition", Macromolecules (in press). 


\begin{abstract}
The effect of pressure on poly(propylene oxide) (PPO) relaxation dynamics near the glass transition has been determined using photon correlation spectroscopy. Experimental results are reported for pressures ranging from $2.5-3.9 \mathrm{kbar}$ at $253 \mathrm{~K}$. The structural relaxation exhibits two distinct processes separated by approximately three orders of magnitude in time. This observation is consistent with results of dipole relaxation spectroscopic studies of PPO. The faster relaxation is associated with local segmental motions important in the glass-rubber transition. The activation volume of this transition suggests that approximately two monomer units are involved. The mean relaxation time for the faster process exhibits an exponential dependence on pressure. The slower relaxation process may be related to self-diffusion of the chain molecules (i.e. normal-mode relaxation of these presumably Rouse-like chains).
\end{abstract}

3. "Conformational and Dynamic Properties of Polymer Loops and Their Mixtures at an Impenetrable Interface", H. S. Gulati, D. C. Driscoll, R. J. Spontak and C. K. Hall, Proc. of Mat. Res. Soc., Boston (1996), (in press).

\begin{abstract}
In this study we investigate the equilibrium conformational properties and dynamic relaxation behavior of polymer loops grafted at an interface using the discontinuous molecular dynamics simulation technique. Differences and similarities between the structural and dynamic properties of polymer loops and tails are identified. The conformational properties of mixtures of polymers loops and tails are also studied using the bond-fluctuation method. The effect of mixture composition on the conformational properties of the individual components in the mixture is discussed.
\end{abstract}

\title{
3. Papers Submitted
}

1. S. Sunderrajan, C.K. Hall and B.D. Freeman, "Sorption Isotherms for Spherical Penetrants in Facilitating Polymeric Media using Monte Carlo Simulations", Mol. Phys. (submitted).

\begin{abstract}
Grand canonical ensemble Monte Carlo (GCMC) simulations of penetrant sorption in polymeric media have been used to predict sorption isotherms as a function of polymerpenetrant interactions and temperature. The polymer is modeled as a collection of hard chains and as a collection of square well chains, while the penetrant is modeled as hard spheres and as square well spheres. Partition coefficients have been compared for the different potentials of interaction for stationary and moving polymeric media, with the stationary polymeric media modeling glassy polymers and the moving polymeric media modeling rubbery polymers. Partition coefficients are found to increase with increasing reservoir penetrant pressure and to be higher in moving polymeric media than in stationary polymeric media. Partitioning was also studied as a function of concentration and relative
\end{abstract}


strength of facilitating sites which have an increased affinity for penetrants. Partition coefficients increased with increasing concentration of facilitating sites and with increasing strength of attraction of facilitating sites for penetrants. The presence of a second penetrant species of normal affinity is found to affect the partitioning of species with a special affinity for facilitating sites. The partition coefficient for the penetrant species with special affinity for facilitating sites is lower in a mixture than when present as the only component. The partition coefficient of the penetrant species of normal affinity at low to moderate pressures and intermediate mole fractions, is higher in a mixture than when present as the only component.

2. S. Sunderrajan, C.K. Hall and B.D. Freeman, "Chemical Potential Gradient Driven Permeation of Small Molecules Through Polymeric Media”, J. Chem. Phys. (submitted).

\begin{abstract}
Grand canonical molecular dynamics techniques are used to study small molecular penetrant permeation through polymeric media. Penetrants are modeled as hard spheres and square well spheres. Polymer is modeled as a collection of hard chains and square well chains. Glassy polymers are modeled using stationary chains while rubbery polymers are modeled using mobile chains. Facilitated transport polymers are also modeled by varying the square well depth for specific sites along polymer chains. Penetrant partitioning, mutual diffusivity, solubility, and permeability are calculated as a function of reservoir chemical potential, barrier packing fraction, and barrier chain length for a variety of penetrant/polymer combinations. Penetrant mutual diffusivity is a function of penetrant mobility in the polymer which, in turn, is a function of available free volume. Penetrant solubility is a function of penetrant/polymer interactions and available free volume. Penetrant permeability is the product of diffusivity and solubility. Penetrant diffusivity and penetrant solubility in a facilitating polymer are functions of the concentration of 'supersites', strength of penetrant/'super-site' interaction, and polymer mobility. In contrast to experimental observations, penetrant permeability through a facilitating polymer is always lower than that through a polymer with no facilitating sites. Possible explanations for this discrepancy are discussed.
\end{abstract}




\section{References}

[1] Dickman, R. and Hall, C. K. , J. Chem. Phys., 85, 4108 (1986).

[2] Honnell, K. G. and Hall, C. K. , J. Chem. Phys., 90, 1841 (1989).

[3] Hall, C. K. , Denlinger, M. A. , and Honnell, K. G. , Fluid Phase Equil., 53, 151 (1989).

[4] Smith, S. W. , Hall, C. K. , and Freeman, B. D. , J. Chem. Phys., 102, 1057 (1995).

[5] Alder, B. J. , Gass, D. M. , and Wainwright, T. E. , J. Chem. Phys., 53, 3813 (1970).

[6] Costa, L. A. , Zhou, Y. , Hall, C. , and Carra, S. , J. Chem. Phys., 102, 6212 (1995).

[7] Sunderrajan, S., Hall, C. K. , and Freeman, B. D. , J. Chem. Phys., 105, 1621 (1996).

[8] Papadopoulou, A. , Becker, E. D. , Lupkowski, M. , and van Swol, F. ., J. Chem. Phys., 98, 4897 (1993).

[9] Heffelfinger, G. S. and van Swol, F. , J. Chem. Phys., 100, 7548 (1994).

[10] Darken, L. S. , Trans. Am. Inst. Mech. Engin., 175, 184 (1948).

[11] Karger, J. and Ruthven, D. , Diffusion in zeolites and other microporous solids. WileyInterscience, New York, (1992).

[12] Sunderrajan, S., Hall, C. K. , and Freeman, B. D. , Mol. Phys., (submitted).

[13] Sunderrajan, S. , Hall, C. K. , and Freeman, B. D. , J. Chem. Phys., (submitted).

[14] Zhou, Y. , Hall, C. K. , and Karplus, M. , Phys. Lett. A, 77, 2822.

[15] Zhou, Y. , Karplus, M. , Wichert, J. , and Hall, C. , (in preparation).

[16] Ptitsyn, O. B. , Protein Chem., 47, 83 (1995).

[17] Schulz, G. E. and Schirmer, R. H. , Principles of protein structure. Springer-Verlag, New York, (1979).

[18] Dill, K. A. and Stigter, D. , Adv. Protein Chem., 46, 59 (1995).

[19] Westaway, D. , Proc. Nat'l Acad. Sci. USA, 91, 6418 (1994). 